\title{
Sharp Wilker-type inequalities with applications
}

Zhen-Hang Yang and Yu-Ming Chu*

${ }^{\text {*Correspondence: }}$

chuyuming2005@126.com

School of Mathematics and

Computation Science, Hunan City

University, Yiyang, 413000, China

\section{Abstract}

In this paper, we prove that the Wilker-type inequality

$$
\frac{2}{k+2}\left(\frac{\sin x}{x}\right)^{k p}+\frac{k}{k+2}\left(\frac{\tan x}{x}\right)^{p}>(<) 1
$$

holds for any fixed $k \geq 1$ and all $x \in(0, \pi / 2)$ if and only if $p>0$ or $p \leq-\frac{\ln (k+2)-\ln 2}{k(\ln \pi-\ln 2)}$ $\left(-\frac{12}{5(k+2)} \leq p<0\right)$, and the hyperbolic version of Wilker-type inequality

$$
\frac{2}{k+2}\left(\frac{\sinh x}{x}\right)^{k p}+\frac{k}{k+2}\left(\frac{\tanh x}{x}\right)^{p}>(<) 1
$$

holds for any fixed $k \geq 1(<-2)$ and all $x \in(0, \infty)$ if and only if $p>0$ or $p \leq-\frac{12}{5(k+2)}$ $\left(p<0\right.$ or $\left.p \geq-\frac{12}{5(k+2)}\right)$. As applications, several new analytic inequalities are presented. MSC: $26 \mathrm{D0} 5 ; 33 \mathrm{~B} 10$

Keywords: Wilker inequality; trigonometric function; hyperbolic function

\section{Introduction}

Wilker [1] proposed two open problems, the first of which states that the inequality

$$
\left(\frac{\sin x}{x}\right)^{2}+\frac{\tan x}{x}>2
$$

holds for all $x \in(0, \pi / 2)$. Inequality (1.1) was proved by Sumner et al. in [2].

Recently, the Wilker inequality (1.1) and its generalizations, improvements, refinements and applications have attracted the attention of many mathematicians (see [3-17] and related references therein).

In [9], Wu and Srivastava established the following Wilker-type inequality:

$$
\left(\frac{x}{\sin x}\right)^{2}+\frac{x}{\tan x}>2 \quad \text { for } x \in(0, \pi / 2)
$$

and its weighted and exponential generalization.

Theorem Wu ([9, Theorem 1]) Let $\lambda>0, \mu>0$ and $p \leq 2 q \mu / \lambda$. If $q>0$ or $q \leq$ $\min (-1,-\lambda / \mu)$, then the inequality

@2014 Yang and Chu; licensee Springer. This is an Open Access article distributed under the terms of the Creative Commons Attribution License (http://creativecommons.org/licenses/by/2.0), which permits unrestricted use, distribution, and reproduction in any medium, provided the original work is properly cited. 


$$
\frac{\lambda}{\lambda+\mu}\left(\frac{\sin x}{x}\right)^{p}+\frac{\mu}{\lambda+\mu}\left(\frac{\tan x}{x}\right)^{q}>1
$$

holds for $x \in(0, \pi / 2)$.

As an application of inequality (1.3), an open problem was proposed, answered and improved by Sándor and Bencze in [18]. Recently, inequality (1.3) and its related inequalities in [9] were extended to Bessel functions [3], and the hyperbolic version of Theorem Wu was presented in [12].

In 2009, Zhu [16] gave another exponential generalization of Wilker inequality (1.1) as follows.

Theorem Zh1 ([16, Theorems 1.1 and 1.2]) Let $0<x<\pi / 2$. Then the inequalities

$$
\left(\frac{\sin x}{x}\right)^{2 p}+\left(\frac{\tan x}{x}\right)^{p}>\left(\frac{x}{\sin x}\right)^{2 p}+\left(\frac{x}{\tan x}\right)^{p}>2
$$

hold if $p \geq 1$, while the first one in (1.4) holds if and only if $p>0$.

Theorem Zh2 ([16, Theorems 1.3 and 1.4]) Let $x>0$. Then the inequalities

$$
\left(\frac{\sinh x}{x}\right)^{2 p}+\left(\frac{\tanh x}{x}\right)^{p}>\left(\frac{x}{\sinh x}\right)^{2 p}+\left(\frac{x}{\tanh x}\right)^{p}>2
$$

hold if $p \geq 1$, while the first one in (1.5) holds if and only if $p>0$.

In [16], Zhu also proposed an open problem: find the respectively largest range of $p$ such that inequalities (1.4) and (1.5) hold. It was solved by Matejička in [19].

Another inequality associated with the Wilker inequality is the following:

$$
2 \frac{\sin x}{x}+\frac{\tan x}{x}>3
$$

for $x \in(0, \pi / 2)$, which is known as the Huygens inequality [20]. The following refinement of Huygens inequality is due to Neuman and Sándor [7]:

$$
2 \frac{\sin x}{x}+\frac{\tan x}{x}>2 \frac{x}{\sin x}+\frac{x}{\tan x}>3
$$

for $x \in(0, \pi / 2)$. Very recently, the generalizations of (1.7) were given by Neuman in [8]. In [21], Zhu proved that the inequalities

$$
\begin{aligned}
& \left(1-\xi_{1}\right) \frac{\sin x}{x}+\xi_{1} \frac{\tan x}{x}>1>\left(1-\eta_{1}\right) \frac{\sin x}{x}+\eta_{1} \frac{\tan x}{x} \\
& \left(1-\xi_{2}\right) \frac{x}{\sin x}+\xi_{2} \frac{x}{\tan x}>1>\left(1-\eta_{2}\right) \frac{x}{\sin x}+\eta_{2} \frac{x}{\tan x}
\end{aligned}
$$

hold for all $x \in(0, \pi / 2)$ with the best constants $\xi_{1}=1 / 3, \eta_{1}=0, \xi_{2}=1 / 3, \eta_{2}=1-2 / \pi$. Later, Zhu [15] generalized inequalities (1.8) and (1.9) to the exponential form as follows. 
Theorem Zh3 ([15, Theorems 1.1 and 1.2]) Let $0<x<\pi / 2$. Then we have

(i) If $p \geq 1$, then the double inequality

$$
(1-\lambda)\left(\frac{x}{\sin x}\right)^{p}+\lambda\left(\frac{x}{\tan x}\right)^{p}<1<(1-\eta)\left(\frac{x}{\sin x}\right)^{p}+\eta\left(\frac{x}{\tan x}\right)^{p}
$$

holds if and only if $\eta \leq 1 / 3$ and $\lambda \geq 1-(2 / \pi)^{p}$.

(ii) If $0 \leq p \leq 4 / 5$, then double inequality (1.10) holds if and only if $\lambda \geq 1 / 3$ and $\eta \leq 1-(2 / \pi)^{p}$.

(iii) If $p<0$, then the second inequality in (1.10) holds if and only if $\eta \geq 1 / 3$.

The hyperbolic version of inequalities (1.7) was given in [7] by Neuman and Sándor. Later, Zhu showed the following.

Theorem Zh4 ([17, Theorem 4.1]) Let $x>0$. Then one has

(i) If $p \geq 4 / 5$, then the double inequality

$$
(1-\lambda)\left(\frac{x}{\sinh x}\right)^{p}+\lambda\left(\frac{x}{\tanh x}\right)^{p}<1<(1-\eta)\left(\frac{x}{\sinh x}\right)^{p}+\eta\left(\frac{x}{\tanh x}\right)^{p}
$$

holds if and only if $\eta \geq 1 / 3$ and $\lambda \leq 0$.

(ii) If $p<0$, then the inequality

$$
(1-\eta)\left(\frac{x}{\sinh x}\right)^{p}+\eta\left(\frac{x}{\tanh x}\right)^{p}>1
$$

holds if and only if $\eta \leq 1 / 3$.

The main aim of this paper is to present the best possible parameter $p$ such that the inequalities

$$
\begin{aligned}
& \frac{2}{k+2}\left(\frac{\sin x}{x}\right)^{k p}+\frac{k}{k+2}\left(\frac{\tan x}{x}\right)^{p}>1 \text { for } x \in(0, \pi / 2), \\
& \frac{2}{k+2}\left(\frac{\sinh x}{x}\right)^{k p}+\frac{k}{k+2}\left(\frac{\tanh x}{x}\right)^{p}>1 \text { for } x \in(0, \infty)
\end{aligned}
$$

or their reversed inequalities hold for certain fixed $k$ with $k(k+2) \neq 0$. As applications, we also present several new analytic inequalities.

\section{Lemmas}

In order to establish our main results, we need several lemmas, which we present in this section.

Lemma 1 Let $A, B$ and $C$ be defined on $(0, \pi / 2)$ by

$$
\begin{aligned}
& A=A(x)=\cos x(\sin x-x \cos x)^{2}(x-\cos x \sin x), \\
& B=B(x)=(x-\cos x \sin x)^{2}(\sin x-x \cos x), \\
& C=C(x)=\sin ^{2} x\left(-2 x^{2} \cos x+x \sin x+\cos x \sin ^{2} x\right) .
\end{aligned}
$$


Then, for fixed $k \geq 1$, the function $x \mapsto C(x) /(k A(x)+B(x))$ is increasing on $(0, \pi / 2)$. Moreover, we have

$$
\frac{5}{12(k+2)}<\frac{C(x)}{k A(x)+B(x)}<1
$$

Proof We clearly see that $A, B>0$ for $x \in(0, \pi / 2)$ because of $\sin x-x \cos x>0$ and $x-$ $\cos x \sin x=(2 x-\sin 2 x) / 2>0$, and $C>0$ because of

$$
\left(-2 x^{2} \cos x+x \sin x+\cos x \sin ^{2} x\right)=x^{2} \cos x\left(\left(\frac{\sin x}{x}\right)^{2}+\frac{\tan x}{x}-2\right)>0
$$

by Wilker inequality (1.1).

Let $D=(k A+B) / C$, then simple computations lead to

$$
\begin{aligned}
D(x) & =\frac{x \sin ^{2} x\left(-2 x^{2} \cos x+x \sin x+\cos x \sin ^{2} x\right)}{(\sin x-x \cos x)(x-\cos x \sin x)\left(\left(1-k \cos ^{2} x\right) x+(k-1) \cos x \sin x\right)} \\
& =\frac{-2 x^{2} \cos x+x \sin x+\cos x \sin ^{2} x}{(\sin x-x \cos x)(x-\cos x \sin x)} \times \frac{x \sin ^{2} x}{k(\sin x-x \cos x) \cos x+(x-\cos x \sin x)} \\
& :=D_{1}(x) \times D_{2}(x) .
\end{aligned}
$$

It follows from [16, Lemma 2.9] that the function $D_{1}$ is positive and increasing on $(0, \pi / 2)$. Hence it remains to prove that the function $D_{2}$ is also positive and increasing. Clearly, $D_{2}(x)>0$, we only need to show that $D_{2}^{\prime}(x)>0$ for $x \in(0, \pi / 2)$. Indeed,

$$
\begin{aligned}
D_{2}^{\prime}(x) & =(k-1) \sin x \frac{\left(-2 x^{2} \cos x+\cos x \sin ^{2} x+x \sin x\right)}{(k(\sin x-x \cos x) \cos x+(x-\cos x \sin x))^{2}} \\
& =\frac{(k-1) x^{2} \sin x \cos x}{(k(\sin x-x \cos x) \cos x+(x-\cos x \sin x))^{2}}\left(\left(\frac{\sin x}{x}\right)^{2}+\frac{\tan x}{x}-2\right),
\end{aligned}
$$

which is clearly positive due to Wilker inequality (1.1). Therefore, $C /(k A+B)$ is increasing on $(0, \pi / 2)$, and

$$
\frac{5}{12(k+2)}=\lim _{x \rightarrow 0} \frac{C(x)}{k A(x)+B(x)}<D(x)<\lim _{x \rightarrow \pi / 2^{-}} \frac{C(x)}{k A(x)+B(x)}=1 .
$$

This completes the proof.

Lemma 2 Let $E, F$ and $G$ be defined on $(0, \infty)$ by

$$
\begin{aligned}
& E=E(x)=\cosh x(\sinh x-x \cosh x)^{2}(x-\cosh x \sinh x), \\
& F=F(x)=(\sinh x-x \cosh x)(x-\cosh x \sinh x)^{2}, \\
& G=G(x)=x \sinh ^{2} x\left(2 x^{2} \cosh x-x \sinh x-\cosh x \sinh ^{2} x\right) .
\end{aligned}
$$

Then, for fixed $k \geq 1(k<-2)$, the function $x \mapsto G(x) /(k E(x)+F(x))$ is decreasing (increasing) on $(0, \infty)$. Moreover, we have

$$
\min \left(0, \frac{12}{5(k+2)}\right)<\frac{G(x)}{k E(x)+F(x)}<\max \left(0, \frac{12}{5(k+2)}\right) .
$$


Proof It is easy to verify that $E, F<0$ for $x \in(0, \infty)$ due to

$$
\begin{aligned}
& (x-\cosh x \sinh x)=(2 x-\sinh 2 x) / 2<0, \\
& (\sinh x-x \cosh x)=x\left(\frac{\sinh x}{x}-\cos x\right)<0 .
\end{aligned}
$$

While $G<0$ because of

$$
\left(2 x^{2} \cosh x-x \sinh x-\cosh x \sinh ^{2} x\right)=-x^{2} \cosh x\left(\left(\frac{\sinh x}{x}\right)^{2}+\frac{\tanh x}{x}-2\right)<0
$$

by Wilker inequality (1.5).

Denote $G /(k E+F)$ by $H$ and simple computations give

$$
\begin{aligned}
H(x) & =\frac{x \sinh ^{2} x\left(2 x^{2} \cosh x-x \sinh x-\cosh x \sinh ^{2} x\right)}{\cosh x(\sinh x-x \cosh x)^{2}(x-\sinh x \cosh x) k+(\sinh x-x \cosh x)(x-\sinh x \cosh x)^{2}} \\
& =\frac{-2 x^{2} \cosh x+x \sinh x+\cosh x \sinh ^{2} x}{(x \cosh x-\sinh x)(\sinh x \cosh x-x)} \times \frac{x \sinh ^{2} x}{(k(x \cosh x-\sinh x) \cosh x+\sinh x \cosh x-x)} \\
& :=H_{1}(x) \times H_{2}(x) .
\end{aligned}
$$

Clearly, $H_{1}(x)>0$, and it was proved in [19, Proof of Lemma 2.2] that $H_{1}$ is decreasing on $(0, \infty)$. In order to prove the monotonicity of $H$, we only need to deal with the sign and monotonicity of $\mathrm{H}_{2}$.

(i) Clearly, $H_{2}(x)>0$ for $k \geq 1$. And we claim that $H_{2}$ is also decreasing on $(0, \infty)$. Indeed,

$$
\begin{aligned}
H_{2}^{\prime}(x) & =-(k-1) \sinh x \frac{\left(-2 x^{2} \cosh x+\cosh x \sinh ^{2} x+x \sinh x\right)}{(x \cosh x-\sinh x)^{2}(\cosh x \sinh x-x)^{2}} \\
& =-\frac{(k-1) x^{2} \sinh x \cosh x}{(x \cosh x-\sinh x)^{2}(\cosh x \sinh x-x)^{2}}\left(\left(\frac{\sinh x}{x}\right)^{2}+\frac{\tanh x}{x}-2\right)<0 .
\end{aligned}
$$

Consequently, $H=H_{1} \times H_{2}$ is positive and decreasing on $(0, \infty)$, and so

$$
0=\lim _{x \rightarrow \infty} \frac{G(x)}{k E(x)+F(x)}<\frac{G(x)}{k E(x)+F(x)}<\lim _{x \rightarrow 0} \frac{G(x)}{k E(x)+F(x)}=\frac{12}{5(k+2)} .
$$

(ii) For $k<-2$, by the previous proof we clearly see that $-H_{2}^{\prime}$ is decreasing on $(0, \infty)$, and so

$$
0<-\frac{1}{k}=\lim _{x \rightarrow \infty}\left(-H_{2}(x)\right)<-H_{2}(x)<\lim _{x \rightarrow 0}\left(-H_{2}(x)\right)=-\frac{3}{k+2},
$$

which implies that $-H_{2}$ is positive and decreasing on $(0, \infty)$, and so is the function $-H=$ $H_{1} \times\left(-H_{2}\right)$. That is, $H$ is negative and increasing on $(0, \infty)$, and inequality $(2.8)$ holds true. This completes the proof.

Remark 1 It should be noted that $k E(x)+F(x)<0$ for $k \geq 1$ and $k E(x)+F(x)>0$ for $k<-2$. In fact, it suffices to notice (2.8) and $G(x)<0$ for $x \in(0, \infty)$. 
Lemma 3 For $k \geq 1$, we have

$$
1>\frac{\ln (k+2)-\ln 2}{k(\ln \pi-\ln 2)}>\frac{12}{5(k+2)} .
$$

Proof It suffices to show that

$$
\begin{aligned}
& \delta_{1}(k)=\frac{\ln (k+2)-\ln 2}{\ln \pi-\ln 2}-k<0, \\
& \delta_{2}(k)=\frac{\ln (k+2)-\ln 2}{\ln \pi-\ln 2}-\frac{12 k}{5(k+2)}>0
\end{aligned}
$$

for $k \geq 1$.

Differentiation gives

$$
\begin{aligned}
& \delta_{1}^{\prime}(k)=\frac{1}{(\ln \pi-\ln 2)(k+2)}-1<0, \\
& \delta_{2}^{\prime}(k)=\frac{1}{5} \frac{5 k+24 \ln 2-24 \ln \pi+10}{(k+2)^{2}(\ln \pi-\ln 2)}>0
\end{aligned}
$$

for $k \geq 1$. Therefore, Lemma 3 follows from $\delta_{1}(k) \leq \delta_{1}(1)=(\ln 3-\ln 2) /(\ln 3-\ln \pi)<0$ and $\delta_{2}(k) \geq \delta_{2}(1)=(\ln 3-\ln 2) /(\ln \pi-\ln 2)-4 / 5>0$.

\section{Main results}

Theorem 1 For fixed $k \geq 1$, inequality (1.13) holds for $x \in(0, \pi / 2)$ if and only if $p>0$ or $p \leq-\frac{\ln (k+2)-\ln 2}{k(\ln \pi-\ln 2)}$.

Proof Inequality (1.13) is equivalent to

$$
f(x)=\frac{2}{k+2}\left(\frac{\sin x}{x}\right)^{k p}+\frac{k}{k+2}\left(\frac{\tan x}{x}\right)^{p}-1>0
$$

for $x \in(0, \pi / 2)$. Differentiation yields

$$
\begin{aligned}
f^{\prime}(x) & =-\frac{2 k p}{k+2} \frac{\sin x-x \cos x}{x^{2}}\left(\frac{\sin x}{x}\right)^{k p-1}+\frac{k p}{k+2} \frac{x-\sin x \cos x}{x^{2} \cos ^{2} x}\left(\frac{\tan x}{x}\right)^{p-1} \\
& =\frac{k p}{k+2} \frac{x-\sin x \cos x}{x^{2} \cos ^{2} x}\left(\frac{\tan x}{x}\right)^{p-1} g(x),
\end{aligned}
$$

where

$$
g(x)=1-4 \frac{\sin x-x \cos x}{2 x-\sin 2 x}\left(\frac{\sin x}{x}\right)^{(k-1) p}(\cos x)^{p+1} .
$$

A simple computation leads to $g\left(0^{+}\right)=0$.

Differentiation again and simplifying give

$$
g^{\prime}(x)=8 \frac{\left(\frac{\sin x}{x}\right)^{(k-1) p}(\cos x)^{p}}{x \sin x(2 x-\sin 2 x)^{2}} h(x),
$$


where

$$
\begin{aligned}
h(x)= & \cos x(\sin x-x \cos x)^{2}(x-\cos x \sin x) k p \\
& +(x-\cos x \sin x)^{2}(\sin x-x \cos x) p \\
& +x \sin ^{2} x\left(-2 x^{2} \cos x+x \sin x+\cos x \sin ^{2} x\right) \\
= & k p A(x)+p B(x)+C(x) \\
= & (k A+B)\left(p+\frac{C}{k A+B}\right),
\end{aligned}
$$

where $A(x), B(x)$ and $C(x)$ are defined as in (2.1), (2.2) and (2.3), respectively.

By (3.2), (3.4) we easily get

$$
\begin{aligned}
& \operatorname{sgn} f^{\prime}(x)=\operatorname{sgn} p \operatorname{sgn} g(x), \\
& \operatorname{sgn} g^{\prime}(x)=\operatorname{sgn} h(x) .
\end{aligned}
$$

Necessity. We first present two limit relations:

$$
\begin{aligned}
\lim _{x \rightarrow 0^{+}} x^{4} f(x) & =\frac{k p}{36}\left(p+\frac{12}{5(k+2)}\right), \\
\lim _{x \rightarrow(\pi / 2)^{-}} f(x) & = \begin{cases}\infty & \text { if } p>0, \\
\frac{2}{k+2}\left(\frac{2}{\pi}\right)^{k p}-1 & \text { if } p<0 .\end{cases}
\end{aligned}
$$

In fact, using power series extension yields

$$
f(x)=\frac{k p}{36} \frac{k p+2 p+12 / 5}{k+2} x^{4}+o\left(x^{4}\right)
$$

which implies the first limit relation (3.8). From the fact that $\lim _{x \rightarrow \pi / 2^{-}} \tan x=\infty$, the second one (3.9) easily follows.

Now we can derive that the necessary condition of (1.13) holds for $x \in(0, \pi / 2)$ from the simultaneous inequalities $\lim _{x \rightarrow 0^{+}} x^{4} f(x) \geq 0$ and $\lim _{x \rightarrow(\pi / 2)^{-}} f(x) \geq 0$. Solving for $p$ yields $p>0$ or

$$
p \leq \min \left(-\frac{12}{5(k+2)},-\frac{\ln (k+2)-\ln 2}{k(\ln \pi-\ln 2)}\right)=-\frac{\ln (k+2)-\ln 2}{k(\ln \pi-\ln 2)},
$$

where the equality holds due to Lemma 3.

Sufficiency. We prove that the condition $p>0$ or $p \leq-\frac{\ln (k+2)-\ln 2}{k(\ln \pi-\ln 2)}$ is sufficient. We divide the proof into three cases.

Case $1 p>0$. Clearly, $h(x)>0$, then $g^{\prime}(x)>0$ and $g(x)>g\left(0^{+}\right)=0$, which together with $\operatorname{sgn} p=1$ yields $f^{\prime}(x)>0$ and $f(x)>f\left(0^{+}\right)=0$.

Case $2 p \leq-1$. By Lemma 1 it is easy to get

$$
p+\frac{C}{k A+B}<p+1 \leq 0
$$

which reveals that $h(x)<0, g^{\prime}(x)<0$ and $g(x)<g\left(0^{+}\right)=0$, which in combination with $\operatorname{sgn} p=-1$ implies $f^{\prime}(x)>0$ and $f(x)>f\left(0^{+}\right)=0$. 
Case $3-1<p \leq-\frac{\ln (k+2)-\ln 2}{k(\ln \pi-\ln 2)}$. Lemma 1 reveals that $\frac{C}{k A+B}$ is increasing on $(0, \pi / 2)$, so is the function $x \mapsto p+\frac{C}{k A+B}:=\lambda(x)$. Since

$$
\lambda\left(0^{+}\right)=p+\frac{12}{5(k+2)}<0, \quad \lambda\left(\frac{\pi}{2}^{-}\right)=p+1>0,
$$

there exists $x_{1} \in(0, \pi / 2)$ such that $\lambda(x)<0$ for $x \in\left(0, x_{1}\right)$ and $\lambda(x)>0$ for $x \in\left(x_{1}, \pi / 2\right)$, and so is $g^{\prime}(x)$. Therefore, $g(x)<g\left(0^{+}\right)=0$ for $x \in\left(0, x_{1}\right)$ but $g\left(\pi / 2^{-}\right)=1$, which implies that there exists $x_{0} \in\left(x_{1}, \pi / 2\right)$ such that $g(x)<0$ for $x \in\left(0, x_{0}\right)$ and $g(x)>0$ for $x \in\left(x_{0}, \pi / 2\right)$. Due to $\operatorname{sgn} p=-1$, it is deduced that $f^{\prime}(x)>0$ for $x \in\left(0, x_{0}\right)$ and $f^{\prime}(x)<0$ for $x \in\left(x_{0}, \pi / 2\right)$, which reveals that $f$ is increasing on $\left(0, x_{0}\right)$ and decreasing on $\left(x_{0}, \pi / 2\right)$. It follows that

$$
\begin{aligned}
& 0=f\left(0^{+}\right)<f(x)<f\left(x_{0}\right)=0 \quad \text { for } x \in\left(0, x_{0}\right) \\
& f\left(x_{0}\right)>f(x)>f\left(\pi / 2^{-}\right)=\frac{2}{k+2}\left(\frac{2}{\pi}\right)^{k p}-1 \geq 0 \quad \text { for } x \in\left(x_{0}, \pi / 2\right)
\end{aligned}
$$

that is, $f(x)>0$ for $x \in(0, \pi / 2)$.

This completes the proof.

Theorem 2 For fixed $k \geq 1$, the reversed inequality of (1.13), that is,

$$
\frac{2}{k+2}\left(\frac{\sin x}{x}\right)^{k p}+\frac{k}{k+2}\left(\frac{\tan x}{x}\right)^{p}<1,
$$

holds for $x \in(0, \pi / 2)$ if and only if $-\frac{12}{5(k+2)} \leq p<0$.

Proof Necessity. If inequality (3.10) holds for $x \in(0, \pi / 2)$, then we have

$$
\lim _{x \rightarrow 0^{+}} \frac{f(x)}{x^{4}}=\frac{k p}{36}\left(p+\frac{12}{5(k+2)}\right) \leq 0 .
$$

Solving the inequality for $p$ yields $-\frac{12}{5(k+2)} \leq p<0$.

Sufficiency. We prove that the condition $-\frac{12}{5(k+2)} \leq p<0$ is sufficient. It suffices to show that $f(x)<0$ for $x \in(0, \pi / 2)$. By Lemma 1 it is easy to get

$$
p+\frac{C}{k A+B} \geq p+\frac{12}{5(k+2)} \geq 0,
$$

which reveals that $h(x)>0, g^{\prime}(x)>0$ and $g(x)>g\left(0^{+}\right)=0$. In combination with $\operatorname{sgn} p=-1$, it implies $f^{\prime}(x)<0$. Thus, $f(x)<f\left(0^{+}\right)=0$, which proves the sufficiency and the proof is completed.

Theorem 3 For fixed $k \geq 1$, inequality (1.14) holds for $x \in(0, \infty)$ if and only if $p>0$ or $p \leq-\frac{12}{5(k+2)}$.

Proof Let

$$
u(x)=\frac{2}{k+2}\left(\frac{\sinh x}{x}\right)^{k p}+\frac{k}{k+2}\left(\frac{\tanh x}{x}\right)^{p}-1 .
$$


Then inequality (1.14) is equivalent to $u(x)>0$. Differentiation leads to

$$
u^{\prime}(x)=-\frac{k p}{2(k+2)} \frac{\sinh 2 x-2 x}{x^{2} \cosh ^{2} x}\left(\frac{\tanh x}{x}\right)^{p-1} v(x),
$$

where

$$
v(x)=1-4 \frac{\sinh x-x \cosh x}{2 x-\sinh 2 x}\left(\frac{\sinh x}{x}\right)^{k p-p}(\cosh x)^{p+1} .
$$

Differentiation again gives

$$
v^{\prime}(x)=\frac{2 \cosh ^{p} x\left(\frac{\sinh x}{x}\right)^{k p-p}}{x \sinh x(x-\cosh x \sinh x)^{2}} w(x)
$$

where

$$
\begin{aligned}
w(x)= & \cosh x(\sinh x-x \cosh x)^{2}(x-\cosh x \sinh x) k p \\
& +(\sinh x-x \cosh x)(x-\cosh x \sinh x)^{2} p \\
& +x \sinh ^{2} x\left(2 x^{2} \cosh x-x \sinh x-\cosh x \sinh ^{2} x\right) \\
= & k p E(x)+p F(x)+G(x)=(k E+F)\left(p+\frac{G}{k E+F}\right),
\end{aligned}
$$

where $E(x), F(x)$ and $G(x)$ are defined as in (2.5), (2.6) and (2.7), respectively.

By (3.12) and (3.14) we easily get

$$
\begin{aligned}
& \operatorname{sgn} u^{\prime}(x)=-\operatorname{sgn} \frac{k}{k+2} \operatorname{sgn} p \operatorname{sgn} v(x), \\
& \operatorname{sgn} v^{\prime}(x)=\operatorname{sgn} w(x) .
\end{aligned}
$$

Necessity. If inequality (1.14) holds for $x \in(0, \infty)$, then we have $\lim _{x \rightarrow 0^{+}} x^{-4} u(x) \geq 0$. Expanding $u(x)$ in power series gives

$$
u(x)=\frac{k}{36} p\left(p+\frac{12}{5 p(k+2)}\right) x^{4}+o\left(x^{4}\right) .
$$

Hence we get

$$
\lim _{x \rightarrow 0^{+}} x^{-4} u(x)=\frac{k}{36} p\left(p+\frac{12}{5(k+2)}\right) \geq 0 .
$$

Solving the inequality for $p$ yields $p>0$ or $p \leq-\frac{12}{5(k+2)}$.

Sufficiency. We prove that the condition $p>0$ or $p \leq-\frac{12}{5(k+2)}$ is sufficient for (1.14) to hold.

If $p>0$, then $w(x)<0$ due to $E, F, G<0$. Hence, from (3.17) we have $v^{\prime}(x)<0$ and $v(x)<$ $\lim _{x \rightarrow 0^{+}} v(x)=0$. It is derived by (3.16) that $u^{\prime}(x)>0$, and so $u(x)>\lim _{x \rightarrow 0^{+}} u(x)=0$. 
If $p \leq-\frac{12}{5(k+2)}$, then by Lemma 2 we have

$$
p+\frac{G}{k E+F} \leq-\frac{12}{5(k+2)}+\frac{G}{k E+F}<0
$$

and

$$
w(x)=(k E+F)\left(p+\frac{G}{k E+F}\right)>0 .
$$

From (3.17) we have $v^{\prime}(x)>0$ and $v(x)>\lim _{x \rightarrow 0^{+}} v(x)=0$. It follows by (3.16) that $u^{\prime}(x)>0$, which implies that $u(x)>\lim _{x \rightarrow 0^{+}} u(x)=0$.

This completes the proof.

Remark 2 For $k \geq 1$, since $\lim _{x \rightarrow \infty} u(x)=\infty$ for $p \neq 0$ and $\lim _{x \rightarrow \infty} u(x)=0$ for $p=0$, there does not exist $p$ such that the reverse inequality of (1.14) holds for all $x>0$. But we can show that there exists $x_{0} \in(0, \infty)$ such that $u(x)<0$, that is, the reverse inequality of $(1.14)$ holds for $-\frac{12}{5(k+2)}<p<0$. The details of the proof are omitted.

Theorem 4 For fixed $k<-2$, the reverse of (1.14), that is,

$$
\frac{2}{k+2}\left(\frac{\sinh x}{x}\right)^{k p}+\frac{k}{k+2}\left(\frac{\tanh x}{x}\right)^{p}<1
$$

holds for $x \in(0, \infty)$ if and only if $p<0$ or $p \geq-\frac{12}{5(k+2)}$.

Proof Necessity. If inequality (3.18) holds for $x \in(0, \infty)$, then we have

$$
\lim _{x \rightarrow 0^{+}} \frac{u(x)}{x^{4}}=\frac{k}{36} p\left(p+\frac{12}{5(k+2)}\right) \leq 0 .
$$

Solving the inequality for $p$ yields $p<0$ or $p \geq-\frac{12}{5(k+2)}$.

Sufficiency. We prove that the condition $p<0$ or $p \geq-\frac{12}{5(k+2)}$ is sufficient for (3.18) to hold.

If $p<0$, then $w(x)=(k E+F)\left(p+\frac{G}{k E+F}\right)<0$ due to $k E+F>0$ and $G<0$. Hence, from (3.17) we have $v^{\prime}(x)<0$ and $v(x)<\lim _{x \rightarrow 0^{+}} v(x)=0$. It is derived by (3.16) that $u^{\prime}(x)<0$, and so $u(x)<\lim _{x \rightarrow 0^{+}} u(x)=0$.

If $p \geq-\frac{12}{5(k+2)}$, then by Lemma 2 we have

$$
p+\frac{G}{k E+F} \geq p+\frac{12}{5(k+2)}>0
$$

and

$$
w(x)=(k E+F)\left(p+\frac{G}{k E+F}\right)>0 .
$$

From (3.17) we have $v^{\prime}(x)>0$ and $v(x)>\lim _{x \rightarrow 0^{+}} v(x)=0$. It follows by (3.16) that $u^{\prime}(x)<0$, which implies that $u(x)<\lim _{x \rightarrow 0^{+}} u(x)=0$.

This completes the proof. 


\section{Applications}

\subsection{Huygens-type inequalities}

Letting $k=1$ in Theorems 1 and 2, we have the following proposition.

Proposition 1 For $x \in(0, \pi / 2)$, the double inequality

$$
\frac{2}{3}\left(\frac{\sin x}{x}\right)^{p}+\frac{1}{3}\left(\frac{\tan x}{x}\right)^{p}>1>\frac{2}{3}\left(\frac{\sin x}{x}\right)^{q}+\frac{1}{3}\left(\frac{\tan x}{x}\right)^{q}
$$

holds if and only if $p>0$ or $p \leq-\frac{\ln 3-\ln 2}{\ln \pi-\ln 2} \approx-0.898$ and $-4 / 5 \leq q<0$.

Let $M_{r}(a, b ; w)$ denote the $r$ th weighted power mean of positive numbers $a, b>0$ defined by

$$
M_{r}(a, b ; w):=\left(w a^{r}+(1-w) b^{r}\right)^{1 / r} \quad \text { if } r \neq 0 \text { and } M_{0}(a, b ; w)=a^{w} b^{1-w}
$$

where $w \in(0,1)$.

Since

$$
\frac{2}{3}\left(\frac{\sin x}{x}\right)^{p}+\frac{1}{3}\left(\frac{\tan x}{x}\right)^{p}=\frac{\frac{2}{3}+\frac{1}{3}(\cos x)^{-p}}{\left(\frac{\sin x}{x}\right)^{-p}}
$$

by Proposition 1 the inequality

$$
\frac{\sin x}{x}>\left(\frac{2}{3}+\frac{1}{3}(\cos x)^{-p}\right)^{-1 / p}=M_{-p}\left(1, \cos x ; \frac{2}{3}\right)
$$

holds for $x \in(0, \pi / 2)$ if and only if $-p \leq 4 / 5$. Similarly, its reversed inequality holds if and only if $-p \geq \frac{\ln 3-\ln 2}{\ln \pi-\ln 2}$. The facts can be stated as a corollary.

Corollary 1 Let $M_{r}(a, b ; w)$ be defined by (4.2). Then, for $x \in(0, \pi / 2)$, the inequalities

$$
M_{\alpha}\left(1, \cos x ; \frac{2}{3}\right)<\frac{\sin x}{x}<M_{\beta}\left(1, \cos x ; \frac{2}{3}\right)
$$

hold if and only if $\alpha \leq 4 / 5$ and $\beta \geq \frac{\ln 3-\ln 2}{\ln \pi-\ln 2} \approx-0.898$.

Remark 3 The Cusa-Huygens inequality [20] refers to

$$
\frac{\sin x}{x}<\frac{2}{3}+\frac{1}{3} \cos x
$$

holds for $x \in(0, \pi / 2)$, which is equivalent to the second inequality in (1.7). As an improvement and generalization, Corollary 1 was proved in [22] by Yang. Here we provide a new proof.

Remark 4 Let $a>b>0$ and let $x=\arcsin \frac{a-b}{a+b} \in(0, \pi / 2)$. Then $\sin x / x=P / A, \cos x=G / A$ and inequalities (4.3) can be rewritten as

$$
M_{\alpha}\left(A, G ; \frac{2}{3}\right)<P<M_{\beta}\left(A, G ; \frac{2}{3}\right),
$$


where $P$ is the first Seiffert mean [23] defined by

$$
P=P(a, b)=\frac{a-b}{2 \arcsin \frac{a-b}{a+b}}
$$

$A$ and $G$ denote the arithmetic and geometric means of $a$ and $b$, respectively.

Let $x=\arctan \frac{a-b}{a+b}$. Then $\sin x / x=T / Q, \cos x=A / Q$, and inequalities (4.3) can be rewritten as

$$
M_{\alpha}\left(Q, A ; \frac{2}{3}\right)<T<M_{\beta}\left(Q, A ; \frac{2}{3}\right)
$$

where $T$ is the second Seiffert mean [24] defined by

$$
T=T(a, b)=\frac{a-b}{2 \arctan \frac{a-b}{a+b}}
$$

$Q$ denotes the quadratic mean of $a$ and $b$.

Obviously, by Corollary 2, the two double inequalities (4.5) (see [22]) and (4.6) hold if and only if $\alpha \leq 4 / 5$ and $\beta \geq \frac{\ln 3-\ln 2}{\ln \pi-\ln 2} \approx-0.898$, (4.6) seems to be a new inequality.

In the same way, taking $k=1$ in Theorem 3 , we get the following.

Proposition 2 For $x \in(0, \infty)$, the inequality

$$
\frac{2}{3}\left(\frac{\sinh x}{x}\right)^{p}+\frac{1}{3}\left(\frac{\tanh x}{x}\right)^{p}>1
$$

holds if and only if $p>0$ or $p \leq-\frac{4}{5}$.

Similar to Corollary 1 , we have the following.

Corollary 2 Let $M_{r}(a, b ; w)$ be defined by (4.2). Then, for $x \in(0, \infty)$, the inequalities

$$
M_{\alpha}\left(1, \cosh x ; \frac{2}{3}\right)<\frac{\sinh x}{x}<M_{\beta}\left(1, \cosh x ; \frac{2}{3}\right)
$$

hold if and only if $\alpha \leq 0$ and $\beta \geq 4 / 5$.

Remark 5 Let $a>b>0$ and $x=\ln \sqrt{a / b}$. Then $\sinh x / x=L / G, \cosh x=A / G$, and (4.8) can be rewritten as

$$
M_{\alpha}\left(G, A ; \frac{2}{3}\right)<L<M_{\beta}\left(G, A ; \frac{2}{3}\right),
$$

where $L$ is the logarithmic means of $a$ and $b$ defined by

$$
L=L(a, b)=\frac{a-b}{\ln a-\ln b} .
$$


Making use of $x=\operatorname{arcsinh} \frac{b-a}{a+b}$ yields $\sinh x / x=N S / A$ and $\cosh x=Q / A$, where $N S$ is the Nueman-Sándor mean defined by

$$
N S=N S(a, b)=\frac{a-b}{2 \operatorname{arcsinh} \frac{a-b}{a+b}} .
$$

Thus, (4.8) is equivalent to

$$
M_{\alpha}\left(A, Q ; \frac{2}{3}\right)<N S<M_{\beta}\left(A, Q ; \frac{2}{3}\right) .
$$

Corollary 2 implies that inequalities (4.9) and (4.10) hold if and only if $\alpha \leq 0$ and $\beta \geq 4 / 5$. The second inequality in (4.10) is a new inequality.

Remark 6 It should be pointed out that all inequalities involving $\sin x / x$ and $\cos x$ or $\sinh x / x$ and $\cosh x$ in this paper can be rewritten as the equivalent inequalities for bivariate means mentioned previously. In what follows we no longer mention this.

\subsection{Wilker-Zhu-type inequalities}

Letting $k=2$ in Theorems 1 and 2, we have the following.

Proposition 3 For $x \in(0, \pi / 2)$, the double inequality

$$
\left(\frac{\sin x}{x}\right)^{2 p}+\left(\frac{\tan x}{x}\right)^{p}>2>\left(\frac{\sin x}{x}\right)^{2 q}+\left(\frac{\tan x}{x}\right)^{q}
$$

holds if and only if $p>0$ or $p \leq-\frac{\ln 2}{2(\ln \pi-\ln 2)} \approx-0.767$ and $-3 / 5 \leq q<0$.

Note that

$$
\frac{\left(\frac{\sin x}{x}\right)^{2 p}+\left(\frac{\tan x}{x}\right)^{p}-2}{\left(\frac{\sin x}{x}\right)^{p}+\frac{\sqrt{8+\cos ^{-2 p} x}+\cos ^{-p} x}{2}}=\left(\frac{x}{\sin x}\right)^{-p}-\frac{\sqrt{8+\cos ^{-2 p} x}-\cos ^{-p} x}{2} .
$$

By Proposition 3 the inequality

$$
\frac{x}{\sin x}>\left(\frac{\sqrt{8+\cos ^{-2 p} x}-\cos ^{-p} x}{2}\right)^{-1 / p}
$$

or

$$
\frac{\sin x}{x}<\left(\frac{\sqrt{8+\cos ^{-2 p} x}+\cos ^{-p} x}{4}\right)^{-1 / p}:=H_{-p}(\cos x)
$$

holds for $x \in(0, \pi / 2)$ if and only if $-p \geq \frac{\ln 2}{2(\ln \pi-\ln 2)}$, where $H_{r}$ is defined on $(0, \infty)$ by

$$
H_{r}(t)=\left(\frac{\sqrt{8+t^{2 r}}+t^{r}}{4}\right)^{1 / r} \quad \text { if } r \neq 0 \text { and } H_{0}(t)=\sqrt[3]{t}
$$

Likewise, its reversed inequality holds if and only if $-p \leq 3 / 5$. This result can be stated as a corollary. 
Corollary 3 Let $H_{r}(t)$ be defined by (4.12). Then, for $x \in(0, \pi / 2)$, the inequalities

$$
H_{\alpha}(\cos x)<\frac{\sin x}{x}<H_{\beta}(\cos x)
$$

are true if and only if $\alpha \leq 3 / 5$ and $\beta \geq \frac{\ln 2}{2(\ln \pi-\ln 2)} \approx 0.767$.

Taking $k=2$ in Theorem 3, we have the following.

Proposition 4 For $x \in(0, \infty)$, the inequality

$$
\left(\frac{\sinh x}{x}\right)^{2 p}+\left(\frac{\tanh x}{x}\right)^{p}>2
$$

holds if and only if $p>0$ or $p \leq-3 / 5$.

In a similar way, we get Corollary 4 .

Corollary 4 Let $H_{r}(t)$ be defined by (4.12). Then, for $x \in(0, \infty)$, the inequalities

$$
H_{\alpha}(\cosh x)<\frac{\sinh x}{x}<H_{\beta}(\cosh x)
$$

are true if and only if $\alpha \leq 0$ and $\beta \geq 3 / 5$.

Now we give a generalization of inequalities (1.4) given by Zhu [15].

Proposition 5 For fixed $k \geq 1$, both chains of inequalities

$$
\begin{aligned}
\frac{2}{k+2}\left(\frac{\sin x}{x}\right)^{k p}+\frac{k}{k+2}\left(\frac{\tan x}{x}\right)^{p} & \geq \frac{k}{k+2}\left(\frac{\sin x}{x}\right)^{k p}+\frac{2}{k+2}\left(\frac{\tan x}{x}\right)^{p} \\
& >\frac{2}{k+2}\left(\frac{x}{\sin x}\right)^{k p}+\frac{k}{k+2}\left(\frac{x}{\tan x}\right)^{p}>1, \\
\frac{2}{k+2}\left(\frac{\sin x}{x}\right)^{k p}+\frac{k}{k+2}\left(\frac{\tan x}{x}\right)^{p} & >\frac{2}{k+2}\left(\frac{x}{\tan x}\right)^{p}+\frac{k}{k+2}\left(\frac{x}{\sin x}\right)^{k p} \\
& \geq \frac{2}{k+2}\left(\frac{x}{\sin x}\right)^{k p}+\frac{k}{k+2}\left(\frac{x}{\tan x}\right)^{p}>1
\end{aligned}
$$

hold for $x \in(0, \pi / 2)$ if and only if $k \geq 2$ and $p \geq \frac{\ln (k+2)-\ln 2}{k(\ln \pi-\ln 2)}$.

Proof The first inequality in (4.15) is equivalent to

$$
\begin{aligned}
& \frac{2}{k+2}\left(\frac{\sin x}{x}\right)^{k p}+\frac{k}{k+2}\left(\frac{\tan x}{x}\right)^{p}-\frac{k}{k+2}\left(\frac{\sin x}{x}\right)^{k p}-\frac{2}{k+2}\left(\frac{\tan x}{x}\right)^{p} \\
& =\frac{k-2}{k+2}\left(\left(\frac{\tan x}{x}\right)^{p}-\left(\frac{\sin x}{x}\right)^{k p}\right)>0 .
\end{aligned}
$$

Due to $\frac{\tan x}{x}>1$ and $\frac{\sin x}{x}<1$, it holds for $x \in(0, \pi / 2)$ if and only if

$$
(k, p) \in\{k \geq 2, p>0\} \cup\{1 \leq k \leq 2, p<0\}:=\Omega_{1} .
$$


The second one is equivalent to

$$
\frac{\frac{k}{k+2}\left(\frac{\sin x}{x}\right)^{k p}+\frac{2}{k+2}\left(\frac{\tan x}{x}\right)^{p}}{\frac{2}{k+2}\left(\frac{x}{\sin x}\right)^{k p}+\frac{k}{k+2}\left(\frac{x}{\tan x}\right)^{p}}>1
$$

which can be simplified to

$$
\left(\frac{\sin x}{x}\right)^{k p}\left(\frac{\tan x}{x}\right)^{p}=\left(\left(\frac{\sin x}{x}\right)^{k+1} \frac{1}{\cos x}\right)^{p}>1 .
$$

It is true for $x \in(0, \pi / 2)$ if and only if $(k, p) \in\{k+1 \geq 3, p \geq 0\}:=\Omega_{2}$.

By Theorem 1, the third one in (4.15) holds for $x \in(0, \pi / 2)$ if and only if

$$
(k, p) \in\{k \geq 1,-p>0\} \cup\left\{k \geq 1,-p \leq-\frac{\ln (k+2)-\ln 2}{k(\ln \pi-\ln 2)}\right\}:=\Omega_{3} .
$$

Hence, inequalities (4.15) hold for $x \in(0, \pi / 2)$ if and only if

$$
(k, p) \in \Omega_{1} \cap \Omega_{2} \cap \Omega_{3}=\left\{k \geq 2, p \geq \frac{\ln (k+2)-\ln 2}{k(\ln \pi-\ln 2)}\right\},
$$

which proves (4.15).

In the same way, we can prove (4.16), the details are omitted.

Letting $k=2$ in Proposition 5, we have the following.

Corollary 5 For $x \in(0, \pi / 2)$, inequality (1.4) holds if and only if $p \geq \frac{\ln 2}{2(\ln \pi-\ln 2)} \approx 0.767$.

Similarly, using Theorem 3 we easily prove the following proposition.

Proposition 6 For fixed $k \geq 1$, the inequalities

$$
\frac{k}{k+2}\left(\frac{\sinh x}{x}\right)^{k p}+\frac{2}{k+2}\left(\frac{\tanh x}{x}\right)^{p}>\frac{2}{k+2}\left(\frac{x}{\sinh x}\right)^{k p}+\frac{k}{k+2}\left(\frac{x}{\tanh x}\right)^{p}>1
$$

hold for $x \in(0, \infty)$ if and only if $k \geq 2$ and $p \geq \frac{12}{5(k+2)}$.

Letting $k=2$ in Proposition 6, we have the following.

Corollary 6 For $x \in(0, \infty)$, inequality (1.5) holds if and only if $p \geq 3 / 5$.

Remark 7 Clearly, Corollaries 5 and 6 offer another method for solving the problems posed by Zhu in [16].

\subsection{Other Wilker-type inequalities}

Taking $k=3,4$ in Theorems 1 and 2, we obtain the following.

Proposition 7 For $x \in(0, \pi / 2)$, the inequality

$$
\frac{2}{5}\left(\frac{\sin x}{x}\right)^{3 p}+\frac{3}{5}\left(\frac{\tan x}{x}\right)^{p}>1
$$


holds if and only if $p>0$ or $p \leq-\frac{\ln 5-\ln 2}{3(\ln \pi-\ln 2)} \approx-0.676$. It is reversed if and only if $-12 / 25 \leq$ $p<0$.

Proposition 8 For $x \in(0, \pi / 2)$, the inequality

$$
\frac{1}{3}\left(\frac{\sin x}{x}\right)^{4 p}+\frac{2}{3}\left(\frac{\tan x}{x}\right)^{p}>1
$$

holds if and only if $p>0$ or $p \leq-\frac{\ln 3}{4(\ln \pi-\ln 2)} \approx-0.608$. It is reversed if and only if $-2 / 5 \leq p<0$.

Putting $k=-3,-4$ in Theorem 3 , we get the following.

Proposition 9 For $x \in(0, \infty)$, the inequality

$$
\left(\frac{\tanh x}{x}\right)^{p}<\frac{2}{3}\left(\frac{x}{\sinh x}\right)^{3 p}+\frac{1}{3}
$$

holds if and only if $p<0$ or $p \geq 12 / 5$.

Proposition 10 For $x \in(0, \pi / 2)$, the inequality

$$
2\left(\frac{\tanh x}{x}\right)^{p}<\left(\frac{x}{\sinh x}\right)^{4 p}+1
$$

holds if and only if $p<0$ or $p \geq 6 / 5$.

\section{Competing interests}

The authors declare that they have no competing interests.

\section{Authors' contributions}

Z-HY carried out the proof of the Wilker-type inequality and drafted the manuscript. Y-MC provided the main idea and carried out the proof of the hyperbolic version of Wilker-type inequality. All authors read and approved the final manuscript.

\section{Acknowledgements}

The authors would like to express their deep gratitude to the referees for giving many valuable suggestions. The research was supported by the Natural Science Foundation of China under Grants 61374086 and 11171307, and the Natural Science Foundation of Zhejiang Province under Grant LY13A010004.

Received: 6 February 2014 Accepted: 16 April 2014 Published: 06 May 2014

\section{References}

1. Wilker, JB: Problem E3306. Am. Math. Mon. 96(1), 55 (1989)

2. Sumner, JS, Jagers, AA, Vowe, M, Anglesio, J: Inequalities involving trigonometric functions. Am. Math. Mon. 98(3), 264-267 (1991)

3. Baricz, Á, Sándor, J: Extensions of the generalized Wilker inequality to Bessel functions. J. Math. Inequal. 2(3), 397-406 (2008)

4. Chen, C-P, Cheung, W-S: Wilker- and Huygens-type inequalities and solution to Oppenheim's problem. Integral Transforms Spec. Funct. 23(5), 325-336 (2012)

5. Chen, C-P, Cheung, W-S: Sharpness of Wilker and Huygens type inequalities. J. Inequal. Appl. 2012, Article ID 72 (2012)

6. Mortici, C: The natural approach of Wilker-Cusa-Huygens inequalities. Math. Inequal. Appl. 14(3), 535-541 (2011)

7. Neuman, E, Sándor, J: On some inequalities involving trigonometric and hyperbolic functions with emphasis on the Cusa-Huygens, Wilker, and Huygens inequalities. Math. Inequal. Appl. 13(4), 715-723 (2010)

8. Neuman, E: On Wilker and Huygens type inequalities. Math. Inequal. Appl. 15(2), 271-279 (2012)

9. $\mathrm{Wu}, \mathrm{S}-\mathrm{H}$, Srivastava, HM: A weighted and exponential generalization of Wilker's inequality and its applications. Integral Transforms Spec. Funct. 18(7-8), 529-535 (2007) 
10. Wu, S-H, Srivastava, HM: A further refinement of Wilker's inequality. Integral Transforms Spec. Funct. 19(9-10), 757-765 (2008)

11. Wu, S-H: On extension and refinement of Wilker's inequality. Rocky Mt. J. Math. 39(2), 683-687 (2009)

12. Wu, S-H, Debnath, L: Wilker-type inequalities for hyperbolic functions. Appl. Math. Lett. 25(5), 837-842 (2012)

13. Zhang, L, Zhu, L: A new elementary proof of Wilker's inequalities. Math. Inequal. Appl. 11(1), 149-151 (2008)

14. Zhu, L: On Wilker-type inequalities. Math. Inequal. Appl. 10(4), 727-731 (2007)

15. Zhu, L: A source of inequalities for circular functions. Comput. Math. Appl. 58(10), 1998-2004 (2009)

16. Zhu, L: Some new Wilker-type inequalities for circular and hyperbolic functions. Abstr. Appl. Anal. 2009, Article ID 485842 (2009)

17. Zhu, L: Inequalities for hyperbolic functions and their applications. J. Inequal. Appl. 2010, Article ID 130821 (2010)

18. Sándor, J, Bencze, M: On Huygen's trigonometric inequality. RGMIA Res. Rep. Collect. 8(3), Article ID 14 (2005)

19. Matejíčka, L: Note on two new Wilker-type inequalities. Int. J. Open Probl. Comput. Sci. Math. 4(1), 79-85 (2011)

20. Huygens, C: Oeuvres Completes 1888-1940. Sociéte Hollondaise des Science, Haga

21. Zhu, L: Some new inequalities of the Huygens type. Comput. Math. Appl. 58(6), 1180-1182 (2009)

22. Yang, ZH: Sharp bounds for Seiffert mean in terms of weighted power means of arithmetic mean and geometric mean. Math. Inequal. Appl. 17(2), 499-511 (2014)

23. Seiffert, H-J: Werte zwischen dem geometrischen und dem arithmetischen Mittel zweier Zahlen. Elem. Math. 42(4), 105-107 (1987)

24. Seiffert, H-J: Aufgabe $\beta$ 16. Die Wurzel 29, 221-222 (1995)

10.1186/1029-242X-2014-166

Cite this article as: Yang and Chu: Sharp Wilker-type inequalities with applications. Journal of Inequalities and Applications 2014, 2014:166

\section{Submit your manuscript to a SpringerOpen ${ }^{\circ}$ journal and benefit from:}

- Convenient online submission

- Rigorous peer review

- Immediate publication on acceptance

Open access: articles freely available online

- High visibility within the field

- Retaining the copyright to your article 